Rev. Int. Contam. Ambie. 36 (3) 657-666, 2020

https://doi.org/10.20937/RICA.53612

\title{
METALES PESADOS Y BIOMARCADORES DE ESTRÉS OXIDATIVO EN LA ALMEJA Tivela mactroides (Born, 1778)
}

Heavy metals and biomarkers of oxidative stress in trigonal clam Tivela mactroides (Born, 1778)

\section{Edgar ZAPATA-VÍVENES ${ }^{1,3 *}$, Luisa ROJAS-DE ASTUDILLO ${ }^{2}$ y Wladimir RODRÍGUEZ ${ }^{3}$}

\author{
${ }^{1}$ Escuela de Acuicultura y Pesquería, Facultad de Ciencias Veterinarias, Universidad Técnica de Manabí, Ecuador \\ ${ }^{2}$ Departamento de Química, Núcleo de Sucre, Universidad de Oriente, Venezuela \\ ${ }^{3}$ Departamento de Biología, Núcleo de Sucre, Universidad de Oriente, Venezuela \\ *Autor para correspondencia; ezapatavivenes@gmail.com
}

(Recibido: mayo 2019; aceptado: noviembre 2019)

Palabras claves: metalotioneínas, lipoperoxidación, tioles, proteínas, xenobióticos

\section{RESUMEN}

Con el objetivo de cuantificar los niveles de metales pesados ( $\mathrm{Cd}, \mathrm{Pb}, \mathrm{Cu}$ y $\mathrm{Zn}$ ) en el tejido blando de la almeja Tivela mactroides de playa Caicara (Venezuela), así como algunos biomarcadores moleculares de estrés oxidativo en la glándula digestiva de este organismo durante distintas temporadas pluviométricas, se procedió a recolectar de 90 a 100 ejemplares durante agosto y diciembre de 2007 (lluvias moderadas y transición lluvia-sequía, respectivamente), así como en abril y julio de 2008 (sequía y lluvias intensas, respectivamente). Las medidas de metales pesados se realizaron por espectrometría de emisión óptica con plasma acoplado inductivamente, y los contenidos de metalotioneínas $(\mathrm{MT})$, tioles totales $\left(-\mathrm{SH}_{\mathrm{T}}\right)$, tioles libres $\left(-\mathrm{SH}_{\mathrm{L}}\right)$, proteínas totales (PT) y substancias reactivas al ácido tiobarbitúrico (TBARS) se determinaron por métodos colorimétricos. Las concentraciones de $\mathrm{Cd}, \mathrm{Pb}$ y $\mathrm{Cu}$ se incrementaron en los periodos de lluvias y las de $\mathrm{Zn}$ en la transición lluvia-sequía. Los niveles de $\mathrm{Cd}$ y $\mathrm{Pb}$ en $T$. mactroides fueron altos en comparación con almejas provenientes de otras zonas costeras de Venezuela, excediendo los valores establecidos por organizaciones internacionales, especialmente durante el periodo de máxima pluviosidad. Los niveles de $\mathrm{MT}$, $-\mathrm{SH}_{\mathrm{L}}$ y TBARS no mostraron variaciones significativas entre épocas de recolección; sin embargo, los niveles de $-\mathrm{SH}_{\mathrm{T}}$ disminuyeron en el máximo periodo lluvioso. Los resultados de los biomarcadores de estrés oxidativo no mostraron indicios de alteraciones moleculares relacionadas con contaminación; sin embargo, los niveles acumulados de $\mathrm{Cd}$ y $\mathrm{Pb}$ señalan una alarma de disponibilidad e ingreso de xenobióticos de origen antrópico.

Key words: metallothionein, lipoperoxidation, thiols, protein, xenobiotics

\begin{abstract}
To evaluate the levels of heavy metals $(\mathrm{Cd}, \mathrm{Pb}, \mathrm{Cu}$ y $\mathrm{Zn})$ in edible tissue, as well as some molecular biomarkers of oxidative stress, in the digestive gland of the triagonal clam Tivela mactroides from Caicara beach (Venezuela) during different pluviometric seasons, we proceeded to collect between 90 and 100 organisms during August and
\end{abstract}


December 2007 (moderate rain and rain-drought transition, respectively), and April and July 2008 (drought and intense rain, respectively). Heavy metal levels were measured by inductively coupled optical emission spectrometry, while the concentrations of metallothioneins $(\mathrm{MTs})$, total thiols $\left(-\mathrm{SH}_{\mathrm{T}}\right)$, free thiols $\left(-\mathrm{SH}_{\mathrm{F}}\right)$, total proteins $(\mathrm{PT})$, and thiobarbituric acid reactive substances (TBARS) were determined by colorimetric methods. $\mathrm{Cd}, \mathrm{Pb}$ and $\mathrm{Cu}$ concentrations increased in rainfall periods and $\mathrm{Zn}$ in the rain-drought transition. $\mathrm{Cd}$ and $\mathrm{Pb}$ concentrations in T. mactroides were elevated in comparison with clams collected from other Venezuelan coastal areas, exceeding the values suggested by international organizations, especially during the maximal rainfall period. $\mathrm{MT},-\mathrm{SH}_{\mathrm{F}}$ and TBARS concentrations did not show significant variations between collection periods; however, $-\mathrm{SH}_{\mathrm{T}}$ decreased in the period of maximum precipitation. The results of oxidative stress biomarkers did not indicate molecular alterations by contamination; however, accumulated levels of $\mathrm{Cd}$ and $\mathrm{Pb}$ are a signal of alarm of bioavailability and entrance of xenobiotics of anthropogenic origin.

\section{INTRODUCCIÓN}

Los metales pesados forman parte de los elementos constituyentes del agua de mar y los sedimentos de los ecosistemas marino-costeros. Estos elementos son originados por procesos naturales (vulcanización, hidrotermalismo, erosión de rocas) o fuentes antrópicas. La persistencia y biodisponibilidad de estos xenobióticos metálicos en el medio, aun cuando se encuentren en bajas concentraciones, pueden tener consecuencias perjudiciales sobre los organismos (Zapata-Vívenes et al. 2012, Hook et al. 2014), en especial los que se encuentran asociados al sedimento.

Los bivalvos marinos, por ser filtradores, sedentarios y bentónicos, acumulan niveles significativos de metales pesados y otros xenobióticos en sus tejidos, por lo cual han sido considerados especies centinelas para monitoreos ambientales (Wang y Guangyuan 2017, Azizi et al. 2018). La almeja Tivela mactroides es una de las especies más usadas en la detección de efectos de contaminantes en zonas caribeñas (Acosta y Lodeiros 2004a, Alfonso et al. 2005, Sardi et al. 2012). Este bivalvo, que tiene una amplia distribución desde las Indias Occidentales hasta Brasil, habita en playas arenosas de alta energía y desembocaduras de ríos. En la región nororiental de Venezuela presenta una alta tasa de crecimiento y relativa importancia económica (Arrieche y Prieto 2006, Herrera y Bone 2011), siendo explotada de manera artesanal para su consumo fresco y en conservas.

T. mactroides, al igual que otros bivalvos, puede experimentar modificaciones bioquímicas que indican un estado de estrés químico (Sardi et al. 2012). Una de las principales respuestas moleculares o biomarcadores de exposición inducidos por metales pesados incluyen la síntesis de metalotioneínas (MT) y el glutatión reducido (GSH); ambas moléculas contienen grupos sulfhidrilos o tioles (-SH) que son determinantes en la neutralización de la toxicidad de metales pesados (Lemus et al. 2012, Saad et al. 2016). De igual manera, es muy común que en organismos expuestos a concentraciones perjudiciales de metales pesados se puedan encontrar cambios en los niveles de moléculas antioxidantes y daños oxidativos a lípidos de las membranas biológicas (Gagné et al. 2008).

Las MT son proteínas termoestables con baja masa molecular (6-12 KDa), ricas en grupos tioles, que se combinan ávidamente con metales de transición como $\mathrm{Cu}, \mathrm{Zn}, \mathrm{Cd}$ y $\mathrm{Hg}$ a través de enlaces trimercáptidos (Lemus et al. 2016). Además, se ha demostrado que estas proteínas presentan funciones antioxidantes (Zapata-Vívenes y Nusetti 2007, Ruttkay-Nedecky et al. 2013) y contribuyen al desarrollo de los organismos (Mao et al. 2012). Al mismo tiempo, GSH es un tripéptido no proteínico, que a pesar de poseer un grupo tiol, participa eficientemente en la defensa antioxidante (Bennedetti et al. 2015). Estas moléculas evitan la acción de las especies reactivas del oxígeno (ERO) y otros radicales libres sobre la propagación del daño oxidativo de los lípidos en las membranas biológicas (Regoli y Giuliani 2014)

El litoral costero del estado Anzoátegui (Venezuela) presenta una intensa actividad pesquera; sin embargo, posee fuentes potenciales de contaminación por metales pesados y otros xenobióticos debido a su cercanía con el asentamiento del Complejo Petroquímico de la región Nororiental y las escorrentías límnicas del río Neverí, cuyo caudal está influenciado por las estaciones de lluvia y sequía. La persistencia de los metales pesados en los ecosistemas marino-costeros representa un costo para la salud, diversidad y sobrevivencia del componente biótico. En este trabajo se determinaron el contenido corporal de metales pesados de interés ecotoxicológico 
$(\mathrm{Cd}, \mathrm{Pb}, \mathrm{Cu}$ y $\mathrm{Zn})$, y algunos biomarcadores de estrés oxidativo, en $\mathrm{T}$. mactroides recolectado en distintas épocas pluviométricas.

\section{MATERIALES Y MÉTODOS}

\section{Organismos}

Los ejemplares adultos de la almeja T. mactroides (de 30 a $40 \mathrm{~mm}$ de longitud anteroposterior) fueron recolectados manualmente en Playa Caicara, en el área costera del morro de Barcelona hasta el litoral de la costa de Jose (10 $10^{\circ} 09^{\prime} 06^{\prime \prime}-10^{\circ} 09^{\prime} 00^{\prime \prime} \mathrm{N}$ y $64^{\circ}$ 43' 06" $-64^{\circ} 55^{\prime} 00^{\prime}$ "W) (Fig. 1). La zona costera de recolección se encuentra en las cercanías de la planta de fraccionamiento del Complejo Criogénico de Oriente perteneciente a Petroquímica de Petróleo de Venezuela (Pequiven) y de la desembocadura del río Neverí. La abundancia estimada de la población de T. mactroides en Playa Caicara es de $\approx 50.84{\text { ind } \mathrm{m}^{-2}}^{-2}$ (Arrieche y Prieto 2006).

Dos recolecciones quincenales (de 90 a 100 individuos) fueron realizadas durante la época de lluvias moderadas (agosto de 2007, $7.91 \mathrm{~mm}^{3}$ ), la transición del periodo de lluvia a sequía (diciembre de 2007, $6.35 \mathrm{~mm}^{3}$ ), el periodo de sequía (abril de 2008, $4.52 \mathrm{~mm}^{3}$ ) y en el periodo anual de mayor intensidad de lluvias (julio de 2008, $11.99 \mathrm{~mm}^{3}$ ) (INAMEH
2008). Los organismos fueron transportados en cavas isotérmicas al laboratorio para los análisis correspondientes. El tejido blando de 40 ejemplares re colectados en cada periodo ambiental fue removido de sus conchas con un equipo de acero inoxidable para la determinación de metales pesados. Las glándulas digestivas de 48 organismos (12 por cada biomarcador) fueron separadas para la estimación de los biomarcadores moleculares de estrés oxidativo (metalotioneínas, tioles totales y libres y lipoperoxidación).

\section{Metales pesados}

Después de desconchar a los animales, los tejidos blandos en fresco fueron pesados y colocados individualmente en matraces Erlenmeyer de $50 \mathrm{~mL}$ para posteriormente ser secados en un horno a $60^{\circ} \mathrm{C}$ hasta obtener una masa constante. De manera subsecuente, el tejido fue predigerido en $10 \mathrm{~mL}$ de ácido nítrico concentrado durante $12 \mathrm{~h}$. La digestión fue realizada por $3 \mathrm{~h}$ a $80^{\circ} \mathrm{C}$, seguida por $2 \mathrm{~h}$ a $120^{\circ} \mathrm{C}$. Después de enfriarse, se le agregaron $5 \mathrm{~mL}$ de agua desionizada y se filtró utilizando papel de filtro Whatman núm. 42, en un matraz aforado de $25 \mathrm{~mL}$, enrasándose con agua desionizada. Las soluciones fueron analizadas en un espectrómetro de emisión óptica inductivamente acoplado a un plasma (marca Perkin Elmer, modelo óptima 5300 DV) (Rojas et al. 2002). De acuerdo

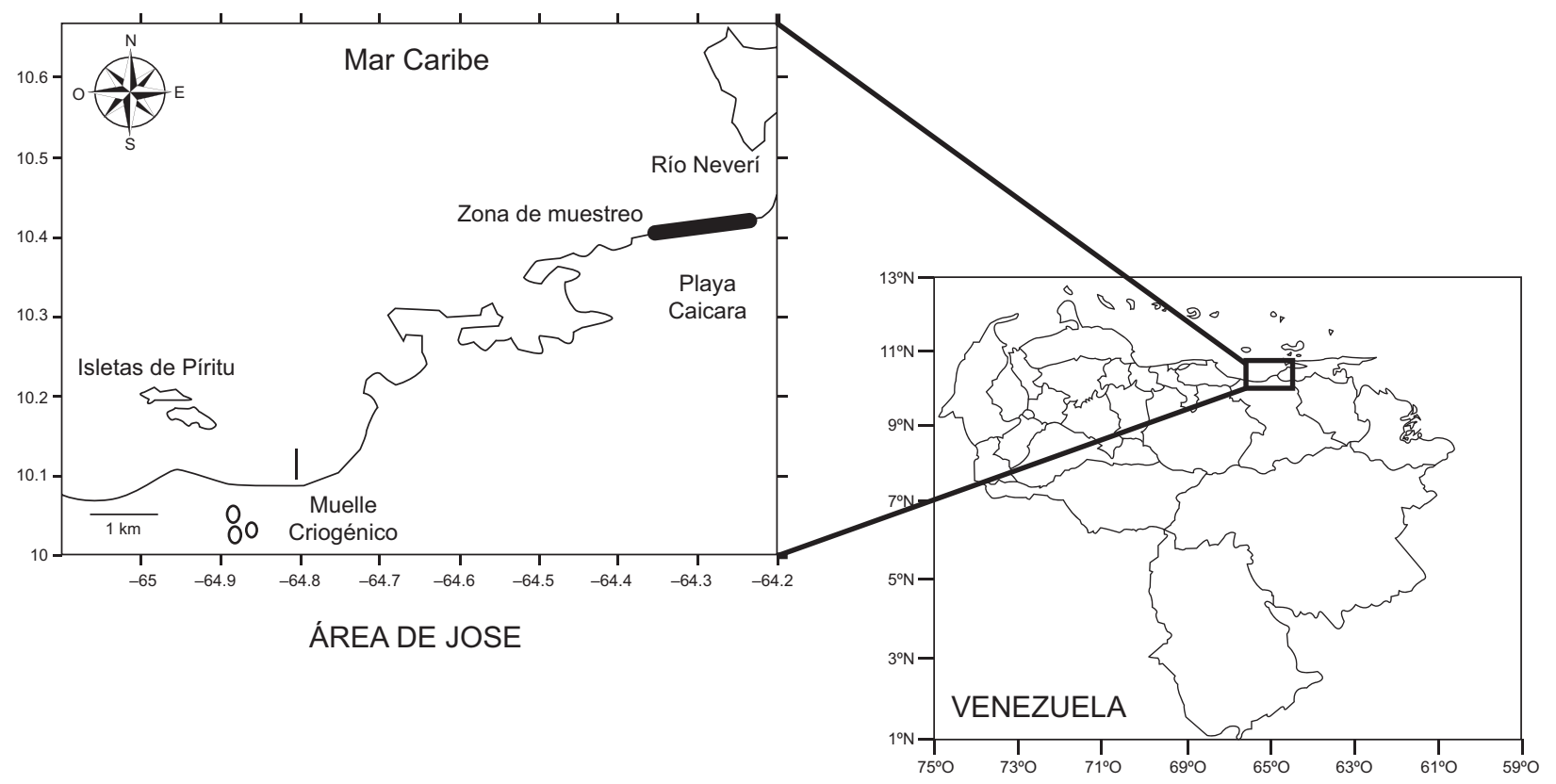

Fig. 1. Localización de la zona de recolección de Tivela mactroides (Playa Caicara, estado Anzoátegui). Área costera del morro de Barcelona y el litoral de la costa de Jose en la región nororiental de Venezuela 
con su importancia ecotoxicológica, se determinaron los niveles de cadmio $(\mathrm{Cd})$, cobre $(\mathrm{Cu})$, plomo $(\mathrm{Pb})$ y zinc ( $\mathrm{Zn})$. Para evaluar la calidad del método de análisis se utilizó un material de referencia certificado de ostras (NIST 1566), el cual se trató de manera similar y se midió por quintuplicado, obteniéndose los porcentajes de recuperación de $104.3 \pm 1.2 \%$ para $\mathrm{Cd}, 93.5 \pm 1.0 \%$ para $\mathrm{Cu}, 91 \pm 2.1 \%$ para $\mathrm{Pb}$ y $98.6 \pm 1.6 \%$ para Zn. Para cada metal, la longitud de onda $(\mathrm{nm})$ y el límite de detección $(\mathrm{mg} / \mathrm{L})$ fueron, respectivamente, 228.80 y 0.009 para Cd; 327.39 y 0.004 para $\mathrm{Cu} ; 220.35$ y 0.007 para $\mathrm{Pb}$ y 206.20 y 0.002 para $\mathrm{Zn}$.

\section{Metalotioneínas}

El contenido de las metalotioneínas (MT) se determinó usando el método espectrofotométrico planteado por Viarengo et al. (1997). Las glándulas digestivas de 12 almejas fueron homogeneizadas individualmente en $4 \mathrm{~mL}$ de solución amortiguadora de extracción Tris-HCl 20 mol/L a pH 8.6, que también contenía $0.5 \mathrm{~mol} / \mathrm{L}$ de sacarosa, $6 \mu \mathrm{mol} / \mathrm{L}$ de leupeptina, $0.5 \mathrm{~mol} / \mathrm{L}$ de fluoruro de fenilmetilsulfonilo, $2 \mathrm{~mol} / \mathrm{L}$ de ditiotreitol y $\beta$ mercaptoetanol $0.01 \%$. El homogenizado se centrifugó a $30000 \mathrm{~g}$ por $20 \mathrm{~min}$. Por cada mililitro de sobrenadante se añadieron $1.05 \mathrm{~mL}$ de etanol y $80 \mu \mathrm{L}$ de cloroformo en frío $\left(-20^{\circ} \mathrm{C}\right)$. Las muestras fueron centrifugadas nuevamente a $6000 \mathrm{~g}$ por $10 \mathrm{~min}$ a $4{ }^{\circ} \mathrm{C}$. El sobrenadante recolectado se combinó con $1 \mathrm{mg}$ de ARN, $40 \mu \mathrm{L}$ de $\mathrm{HCl}$ al $37 \%$ y etanol absoluto frío al $87 \%$. Las muestras se mantuvieron a $-20{ }^{\circ} \mathrm{C}$ por $1 \mathrm{~h}$ y se centrifugaron nuevamente a $6000 \mathrm{~g}$ por $10 \mathrm{~min}$.

El precipitado fue lavado con etanol $87 \%$ y cloroformo $1 \%$ en solución amortiguadora de homogenización. Posteriormente se centrifugó a $6000 \mathrm{~g}$ por 10 min y se secó en una atmósfera de nitrógeno. El precipitado fue resuspendido en $150 \mu \mathrm{L}$ de $\mathrm{NaCl}$ a 0.25 $\mathrm{mol} / \mathrm{L}$ y $150 \mu \mathrm{L}$ de $\mathrm{HCl}$ al $1 \mathrm{~mol} / \mathrm{L}$ que contenía EDTA a $4 \mathrm{~mol} / \mathrm{L}$. Luego se agregaron $4.2 \mathrm{~mL}$ de $\mathrm{NaCl}$ al 2 $\mathrm{mol} / \mathrm{L}$ y ácido 5,5' dithiobis-2-nitrobenzoico (DNBT) al $0.43 \mathrm{mmol} / \mathrm{L}$ en solución amortiguadora de $\mathrm{Na}$ fosfato al $0.2 \mathrm{~mol} / \mathrm{L} \mathrm{a} \mathrm{pH} 8$. Finalmente, la muestra fue centrifugada a $3000 \mathrm{~g}$ por $5 \mathrm{~min}$. El sobrenadante se midió a una longitud de onda de $412 \mathrm{~nm}$, usando como estándar glutatión reducido (GSH Sigma) a $3 \mathrm{mmol} / \mathrm{L}$.

\section{Tioles totales $\left(-\mathrm{SH}_{\mathrm{T}}\right)$}

Se prepararon homogeneizados de cada glándula digestiva (en proporción 1:4 m:v) de 12 organismos por estación, en solución amortiguadora Tris $0.02 \mathrm{~mol} / \mathrm{L}$ con $0.02 \mathrm{~mol} / \mathrm{L} \mathrm{Na}_{2} \mathrm{EDTA}$, pH 8.2 (TRISEDTA), y se dejaron por $5 \mathrm{~h}$ a $8^{\circ} \mathrm{C}$. Posteriormente se realizó una centrifugación de la muestra a $4000 \mathrm{~g}$ y $4{ }^{\circ} \mathrm{C}$ por $10 \mathrm{~min}$. Se utilizaron $40 \mu \mathrm{L}$ del sobrenadante y se le adicionaron $150 \mu \mathrm{L}$ de TRIS-EDTA, $25 \mu \mathrm{l}$ de DTNB y $800 \mu \mathrm{L}$ de metanol. Esta mezcla se centrifugó a $5000 \mathrm{~g}$ durante $5 \mathrm{~min}$, para luego proceder a realizar la lectura a una longitud de onda de $412 \mathrm{~nm}$ (Sedlak y Lindsay 1968) en un espectrofotómetro Genesys 20. Se utilizó como estándar $\mathrm{GSH}$ a $10 \mu \mathrm{mol} / \mathrm{L}$.

\section{Tioles libres $\left(-\mathrm{SH}_{\mathrm{L}}\right)$}

Las glándulas digestivas $(0.25 \mathrm{~g} / \mathrm{mL})$ de 12 individuos por estación se homogenizaron de manera individual en una solución amortiguadora Tris-EDTA $100 \mathrm{mmol} / \mathrm{l} \mathrm{pH} \mathrm{8.9,} \mathrm{para} \mathrm{luego} \mathrm{centrifugarse} \mathrm{a} 2000 \mathrm{~g}$ durante $5 \mathrm{~min}$. Se tomaron $200 \mu \mathrm{L}$ del sobrenadante y se mezclaron con un volumen igual de ácido tricloroacético al $20 \%$ (ATC), incubándose a $-40{ }^{\circ} \mathrm{C}$ durante $1 \mathrm{~h}$. Posteriormente se centrifugaron a $5000 \mathrm{~g}$ durante $15 \mathrm{~min}$. Se tomaron $200 \mu \mathrm{L}$ del sobrenadante y se le agregaron $800 \mu \mathrm{L}$ de Tris $\mathrm{HCl}$ pH 8.9 más 80 $\mu \mathrm{L}$ de DTNB (Ellman 1959). Se realizó la lectura a una longitud de onda de $412 \mathrm{~nm}$ en un espectrofotómetro UV-VIS Pelkin Elmer (modelo lambda 25). Se preparó una curva estándar con GSH a $100 \mu \mathrm{mol} / \mathrm{L}$.

\section{Peroxidación de lípidos}

La peroxidación lipídica se estimó mediante el protocolo propuesto por Ohkawa et al. (1979). Las glándulas digestivas de 12 organismos por estación se homogeneizaron $(50 \mathrm{mg} / \mathrm{mL})$ por separado en una solución amortiguadora de fosfato de sodio a $100 \mathrm{mmol} / \mathrm{L}, \mathrm{pH} 7.5$ y $4{ }^{\circ} \mathrm{C}$. El extracto se centrifugó a $6000 \mathrm{~g}$, a $4{ }^{\circ} \mathrm{C}$ por $20 \mathrm{~min}$. Se incubaron $250 \mu \mathrm{L}$ del homogeneizado en baño de agua a $37^{\circ} \mathrm{C}$ con agitación constante durante $15 \mathrm{~min}$. A cada homogeneizado se le añadieron $250 \mu \mathrm{L}$ de ATC $12.5 \%$, $\mathrm{HCl} 0.8 \mathrm{~mol} / \mathrm{L}$ y $500 \mu \mathrm{L}$ de solución de ácido tiobarbitúrico al $1 \%$. Las muestras fueron colocadas en un baño de agua a $90{ }^{\circ} \mathrm{C}$ con agitación constante por $1 \mathrm{~h}$. Se dejaron a temperatura ambiente por $5 \mathrm{~min}$ y posteriormente se colocaron en baño de hielo por $10 \mathrm{~min}$. Cada muestra se centrifugó a $5000 \mathrm{~g}$ durante 10 min a temperatura ambiente. Las absorbancias de las muestras fueron leídas a una longitud de onda de $535 \mathrm{~nm}$. Se usó como estándar 1,1,3,3-tetraetoxipropano.

\section{Proteínas totales (PT)}

En alícuotas del homogenizado anterior se procedió a determinar la concentración de proteínas totales por el método de Lowry et al. (1951), utilizando como estándar albúmina de suero de bovino. 


\section{Análisis estadístico}

Para establecer diferencias entre los niveles de metales pesados y los biomarcadores moleculares en T. mactroides en las épocas pluviométricas muestreadas se aplicó un análisis no paramétrico KruskallWallis (Sokal y Rohlf 2012), mediante el programa estadístico Statgraphics Plus 5.1.

\section{RESULTADOS}

\section{Metales pesados y biomarcadores}

Las concentraciones de metales pesados estimadas en todo el tejido blando de T. mactroides presentaron variaciones durante las distintas temporadas pluviométricas (Cuadro I). De manera general, los promedios de $\mathrm{Cd}, \mathrm{Pb}$ y $\mathrm{Cu}$ se mostraron más elevados durante los periodos lluviosos y la transición lluvia-sequía, y los menores promedios se observaron en el periodo de sequía. Adicionalmente, en el periodo de máximas lluvias, las concentraciones de $\mathrm{Cd}(16.01 \pm 9.30 \mu \mathrm{g} / \mathrm{g}$ masa seca $)$ y de $\mathrm{Pb}(7.90$ $\pm 5.13 \mu \mathrm{g} / \mathrm{g}$ masa seca), también expresadas en la unidad $\mu \mathrm{g} / \mathrm{g}$ masa fresca $(2.96 \pm 1.52 \mu \mathrm{g} \mathrm{Cd} / \mathrm{g}$ y 2.18 $\pm 1.20 \mu \mathrm{g} \mathrm{Pb} / \mathrm{g}$ ), superaron los límites permisibles recomendados por la organización Mundial de la Salud y la Organización de las Nacionales Unidad para la Alimentación y la Agricultura (FAO/WHO 2018), la Comisión Europea (EC 2017) y la Agencia Nacional de Vigilancia Sanitaria del Brasil (ANVISA 2013). Estas organizaciones sugieren valores de concentraciones límites en tejidos blandos de bivalvos menores a $2.0 \mu \mathrm{g} / \mathrm{g}$ masa fresca para $\mathrm{Cd}$ y por debajo de $1.5 \mu \mathrm{g} / \mathrm{g}$ masa fresca para $\mathrm{Pb}$. El $\mathrm{Cu}$ presentó sus mayores promedios durante ambos periodos lluviosos y la transición lluvia-sequía; y los mayores promedios de $\mathrm{Zn}$ fueron observados durante periodos de lluvias moderadas y la transición lluvia-sequía.

Los niveles de MT, grupos - $\mathrm{SH}_{\mathrm{L}}$, PT y TBARS en la glándula digestiva de T. mactroides no mostraron diferencias significativas durante los distintos periodos pluviométricos examinados. No obstante, las concentraciones de $-\mathrm{SH}_{\mathrm{T}}$ mostraron un descenso significativo en la temporada de máxima pluviosidad (Cuadro II).

CUADRO I. CONCENTRACIÓN DE METALES PESADOS EN EL TEJIDO BLANDO DE T. mactroides DE PLAYA CAICARA, ESTADO ANZOÁTEGUI, VENEZUELA

\begin{tabular}{lcccrc}
\hline Metales & $\begin{array}{c}\text { Lluvias moderadas } \\
(\mu \mathrm{g} / \mathrm{g} \text { masa seca })\end{array}$ & $\begin{array}{c}\text { Transición lluvia- } \\
\text { sequía }(\mu \mathrm{g} / \mathrm{g} \text { masa seca })\end{array}$ & $\begin{array}{c}\text { Sequía } \\
(\mu \mathrm{g} / \mathrm{g} \text { masa seca })\end{array}$ & $\begin{array}{c}\text { Máximo periodo lluvioso } \\
(\mu \mathrm{g} / \mathrm{g} \text { masa seca })\end{array}$ & $\begin{array}{c}\text { Valor P } \\
(\text { Kruskal-Wallis })\end{array}$ \\
\hline $\mathrm{Cd}$ & $2.44 \pm 0.91^{\mathrm{a}}$ & $1.92 \pm 0.97^{\mathrm{a}}$ & $0.35 \pm 0.25^{\mathrm{a}}$ & $16.01 \pm 9.30^{\mathrm{b}}$ & $115.84<0.001$ \\
$\mathrm{~Pb}$ & $3.31 \pm 5.06^{\mathrm{c}}$ & $2.67 \pm 4.08^{\mathrm{bc}}$ & $0.69 \pm 0.39^{\mathrm{ab}}$ & $7.90 \pm 5.13^{\mathrm{d}}$ & $66.48<0.001$ \\
$\mathrm{Cu}$ & $20.30 \pm 7.41^{\mathrm{b}}$ & $15.24 \pm 6.54^{\mathrm{b}}$ & $6.47 \pm 3.01^{\mathrm{a}}$ & $29.44 \pm 21.68^{\mathrm{c}}$ & $58.80<0.001$ \\
$\mathrm{Zn}$ & $115.06 \pm 46.41^{\mathrm{b}}$ & $125.51 \pm 63.60^{\mathrm{b}}$ & $61.91 \pm 15.14^{\mathrm{a}}$ & $66.64 \pm 60.66^{\mathrm{a}}$ & $49.74<0.001$ \\
\hline
\end{tabular}

Los resultados muestran los promedios \pm desviación estándar. Tratamientos con letras desiguales son significativamente diferentes $(\mathrm{p}<0.05)$ y letras iguales indican un valor de $\mathrm{p}>0.05(\mu \mathrm{g} / \mathrm{g}$ masa seca $)$

CUADRO II. NIVELES DE METALOTIONEÍNAS, GRUPOS SULFHIDRILOS TOTALES Y LIBRES, PROTEÍNAS TOTALES Y SUSTANCIAS QUE REACCIONAN AL ÁCIDO TIOBARBITÚRICO EN LA GLÁNDULA DIGESTIVA DE $T$. mactroides DE PLAYA CAICARA, ESTADO ANZOÁTEGUI, VENEZUELA

\begin{tabular}{|c|c|c|c|c|c|}
\hline Biomarcadores & $\begin{array}{l}\text { Lluvias } \\
\text { moderadas }\end{array}$ & $\begin{array}{c}\text { Transición } \\
\text { lluvia-sequía }\end{array}$ & Sequía & $\begin{array}{c}\text { Máximo periodo } \\
\text { lluvioso }\end{array}$ & $\begin{array}{c}\text { Valor P } \\
\text { (Kruskal-Wallis) }\end{array}$ \\
\hline $\mathrm{MT}(\mu \mathrm{g} / \mathrm{g})$ & $52.35 \pm 28.24$ & $40.27 \pm 25.68$ & $40.56 \pm 16.01$ & $64.47 \pm 21.27$ & $6.03>0.05$ \\
\hline$-\mathrm{SH}_{\mathrm{T}}(\mathrm{nmol} / \mathrm{g})$ & $29.57 \pm 6.76^{\mathrm{b}}$ & $37.20 \pm 7.32^{\mathrm{b}}$ & $32.04 \pm 15.95^{\mathrm{b}}$ & $19.66 \pm 4.39^{\mathrm{a}}$ & $13.08<0.01$ \\
\hline$-\mathrm{SH}_{\mathrm{L}}(\mathrm{nmol} / \mathrm{g})$ & $1.52 \pm 0.63$ & $1.17 \pm 0.25$ & $1.12 \pm 0.66$ & $1.18 \pm 0.74$ & $2.79>0.05$ \\
\hline PT $(\mathrm{mg} / \mathrm{g})$ & $252.26 \pm 40.20$ & $214.73 \pm 65.43$ & $241.27 \pm 59.19$ & $227.74 \pm 111.9$ & $1.79>0.05$ \\
\hline TBARS (nmol/mg PT) & $34.57 \pm 8.03$ & $33.26 \pm 5.81$ & $38.38 \pm 9.35$ & $33.61 \pm 8.44$ & $2.56>0.05$ \\
\hline
\end{tabular}

Los resultados muestran los promedios \pm desviación estándar. Tratamientos con letras desiguales son significativamente diferentes $(\mathrm{p}<0.05)$ y letras iguales indican un valor de $\mathrm{p}>0.05$

MT: metalotioneínas, $-\mathrm{SH}_{\mathrm{T}}$ : grupos sulfhidrilos totales, $-\mathrm{SH}_{\mathrm{L}}$ : grupos sulfhidrilos libres, PT: proteínas totales Ys, TBARS: sustancias que reaccionan al ácido tiobarbitúrico 


\section{DISCUSIÓN}

Los niveles de metales pesados detectados en T. mactroides ponen en evidencia su capacidad de acumulación de xenobióticos, especialmente en periodos de alta pluviosidad. Se infiere que el incremento observado en las concentraciones de $\mathrm{Cd}$, $\mathrm{Pb}$ y $\mathrm{Cu}$ en épocas lluviosas se encuentra asociado a la ocurrencia de fenómenos de escurrimiento, así como aumento de las partículas suspendidas en la columna de agua, que pueden favorecer su disponibilidad a la biota. Los periodos de lluvias aumentan el caudal del río Neverí, lo cual aunado a cambios en la hidrodinámica del mar Caribe, pueden contribuir a la disponibilidad de metales pesados de manera estacional. En otras localidades se ha demostrado la incidencia de los cambios ambientales de las temporadas secas y lluviosas sobre la variación del contenido de metales pesados $(\mathrm{Cd}, \mathrm{Pb})$ en distintas especies de bivalvos (Frías-Espericueta et al. 2008, Pinto et al. 2014).

Las concentraciones más elevadas de $\mathrm{Cd}$ en $T$. mactroides registradas en el máximo periodo de lluvias superan a las informadas por Acosta y Lodeiros (2003) para la misma almeja recolectada en Boca de Paparo (estado Miranda, Venezuela) y playa Güiria (estado Sucre, Venezuela), e incluso a los niveles publicados por Alfonso et al. (2005) en localidades de la costa centro-oriental venezolanas. Acosta y Lodeiros (2003) observaron promedios de $\mathrm{Cd}$ de 4.48 $\pm 4.08 \mu \mathrm{g} / \mathrm{g}$ en organismos (con una alta variabilidad intragrupal) recolectados en el mes de julio (mes de inicio de la temporada de lluvia), y encontraron a su vez una relación negativa con los biomarcadores de condición fisiológica de los animales (ARN/ADN). Para la misma especie, Alfonso et al. (2005) encontraron concentraciones de Cd entre 1.02 y $1.53 \mu \mathrm{g} / \mathrm{g}$ en el organismo completo. Estos últimos autores demostraron además similitud en los patrones de variaciones temporales durante todo un año de recolección, observando promedios de concentraciones máximas para $\mathrm{Cd}(4.96 \pm 0.16 \mu \mathrm{g} / \mathrm{g})$ y $\mathrm{Cu}(32.57 \pm$ $0.97 \mu \mathrm{g} / \mathrm{g}$ ) durante los meses de febrero a marzo.

$\mathrm{El} \mathrm{Pb}$ registró un aumento en los organismos durante los periodos lluviosos, posiblemente asociado a la resuspensión del metal contenido en los sedimentos superficiales hacia la columna de agua. La disponibilidad de $\mathrm{Pb}$ en sedimentos de zonas costeras aledañas al sitio de captura de las almejas ha sido demostrada por Bonilla et al. (1995), encontrando concentraciones por encima de lo establecido por las normativas ambientales internacionales. Tales concentraciones están relacionadas con la actividad petroquímica
(García et al. 2008, Croquer et al. 2016). Se sugiere que la acción de las precipitaciones intensas, cambios en el caudal del río Neverí, variaciones del oleaje, vientos y corrientes del Mar Caribe, permitan aumentar la biodisponibilidad de dicho metal en playa Caicara, facilitando así su captación y absorción por parte de las almejas. Al parecer, la presencia de $\mathrm{Pb}$ en T. mactroides es común en zonas costeras de la región nororiental de Venezuela, encontrándose promedios de $\mathrm{Pb}$ de $2.7 \pm 0.4 \mu \mathrm{g} / \mathrm{g}$ (Jaffé et al. 1995) y $1.5 \pm$ $4.9 \mu \mathrm{g} / \mathrm{g}$ y $3.7 \pm 1.3 \mu \mathrm{g} / \mathrm{g}$ (LaBrecque et al. 2004) en organismos recolectados en diversas localidades del estado Miranda; los mismos vinculados a diferente grados de perturbación ambiental.

Los promedios obtenidos para el $\mathrm{Cu}$ muestran similitud con los reportados por Acosta y Lodeiros (2004b) en Río Chico y Boca de Paparo en el estado Miranda y Playa Güiria en el estado Sucre. El Cu es un elemento traza esencial para el crecimiento y desarrollo de los organismos, ya que forma parte de la estructura de oxidasas, hidrolasas, hemocianina, metalotioneínas y otras proteínas (Harris 1991). El ingreso y absorción de $\mathrm{Cu}$ tiene clara relación con su biocinética, la eficacia de asimilación, la tasa de alimentación de los organismos o la entrada de estos metales provenientes de efluentes (Pan y Wang 2009). Algunos investigadores como Bonilla et al. (1995) y LaBrecque et al. (2004) han reportado altos niveles de este metal en sedimentos superficiales, lo que permite la disponibilidad en el medio. Según Fernández et al. (2007), las concentraciones de $\mathrm{Cu}$ en este bivalvo en costas venezolanas varían de 58.9 a $152 \mu \mathrm{g} / \mathrm{g}$ de masa seca, y sus promedios son similares a los encontrados en esta investigación.

El Zn presentó sus mayores concentraciones en $T$. mactroides durante los meses de lluvias moderadas y transición a la sequía. Las concentraciones medidas en este trabajo se encontraron por debajo de las reportadas por Fernández et al. (2007) para la misma especie de almeja (226.0 a $266.0 \mu \mathrm{g} / \mathrm{g}$ de masa seca). La variabilidad de la acumulación corporal de este metal depende de la disponibilidad en el medio y de los requisitos metabólicos de las almejas, pues se sabe que el $\mathrm{Zn}$, al igual que $\mathrm{Cu}$, son indispensables como componentes catalíticos para el crecimiento y reproducción, y su captación tiende a incrementarse con el tiempo de exposición y la talla del cuerpo (Wang y Rainbow 2010, Wang y Guangyuan 2017).

En los bivalvos existen reportes que indican que un alto porcentaje de los metales pesados son absorbidos y acumulados en sus tejidos, con una baja tasa de excreción, pudiendo variar sus concentraciones no sólo con estacionalidad (Salman et al. 2013) y la 
disponibilidad de los metales en el medio (Lemus et al. 2010), sino también con su ciclo reproductivo. La gametogénesis en T. mactroides ocurre entre de marzo a junio (meses de sequía) y su maduración gonadal de julio a noviembre (meses de lluvia), observándose un periodo de desove en diciembre (Prieto et al. 1983, Freites et al. 2014). Se infiere que posiblemente la mayor demanda de alimentos, sustancias complementarias (metales esenciales) y energía para la formación de gametos conlleve a una acelerada captación de metales pesados. A su vez, la relación del contenido de metales pesados respecto a su masa corporal podría notarse ligeramente incrementada en los periodos de desoves.

Además de recibir la influencia del río Neverí, Playa Caicara ha sufrido un proceso lento de contaminación producto de la desviación de algunos colectores de aguas residuales y empotramientos ilegales de aguas servidas en la zona baja del río, donde su cauce converge con la zona urbana. Adicionalmente, presenta una fuente potencial de efluentes de contaminación y otros desechos industriales provenientes de la actividad de refinación del complejo petrolero-petroquímico. De manera general, cantidades significativas de metales pesados pueden ingresar en los ecosistemas marinos vía efluentes domésticos, industriales, descargas de los ríos y emanaciones a la atmosfera, los cuales pueden depositarse y acumularse en el sedimento mediante procesos biogeoquímicos, y posteriormente ingresar en los bivalvos (Wang et al. 2018).

De los marcadores moleculares de estrés oxidativo evaluados, solo - $\mathrm{SH}_{\mathrm{T}}$ exhibió un cambio significativo en la época de máxima pluviosidad, lo cual podría estar asociado con los incrementos observados en las concentraciones de $\mathrm{Cd} \mathrm{y} \mathrm{Pb}$ en $T$. mactroides. Dependiendo de la capacidad de los organismos para activar sus mecanismos de defensa contra xenobióticos, algunos metales pesados propician la utilización de los grupos sulfhidrilos. Se sabe que el Cd también tiene una alta afinidad con el GSH para formar complejos (Lemus et al. 2010), lo cual ocasiona una baja disponibilidad de la molécula a nivel celular; esto puede evitar lesiones oxidativas a nivel de lípidos de las membranas biológicas. Nuestros resultados sugieren que $T$. mactroides posee estrategias celulares que le permiten el almacenaje eficiente de distintos metales pesados sin alterar sus respuestas bioquímicas. Por el contrario, en otras especies de bivalvos el ingreso de metales pesados puede inducir cambios en las moléculas ricas en grupos sulfhidrilos, como la biosíntesis de metalotioneínas, la disminución de los niveles del glutatión (GSH) y la producción de peróxidos lipídicos (Regoli y Guiliani 2014).
En este estudio no se evidenciaron variaciones en los niveles de MT en relación con los cambios estacionales, a pesar de que la asociación del incremento de estas metaloproteínas en periodos de surgencia caribeña se ha señalado en otras especies de bivalvos (Lemus et al. 2016). El metabolismo de los metales está regulado por mecanismos complejos que, en muchos casos, implican un aumento de la concentración de moléculas enlazadoras, ya que acrecientan los niveles de metales en el tejido (Kang 2006, Rabei et al. 2018). Adicionalmente, las MT intervienen en la homeostasis y la desintoxicación de metales, aunque responden a un sinnúmero de efectores y pueden estar involucradas en muchos procesos fisiológicos, e incluso pueden ser inducibles por xenobióticos no metálicos (Wang y Rainbow 2010).

Los marcadores estimados no reflejaron modificaciones en el estatus antioxidante de la glándula digestiva de T. mactroides en relación con la acumulación de metales pesados en el tejido blando completo. Sin embargo, en la época de lluvias intensas las almejas T. mactroides recolectadas en Playa Caicara presentaron elevadas concentraciones de $\mathrm{Cd}$ y $\mathrm{Pb}$ (incluso en su relación $\mu \mathrm{g} / \mathrm{g}$ masa fresca), las cuales se encuentran por arriba a lo establecido por la Organización de las Naciones Unidas para la agricultura y la alimentación, la Comunidad Europea y la Agencia Sanitaria del gobierno Brasileño. Los autores recomiendan el uso de T. mactroides en programas de vigilancia regulada de ambientes marino-costeros, basados sólo en la supervisión continua de disponibilidad de los niveles de metales pesados.

\section{CONCLUSIONES}

A pesar de que los biomarcadores de estrés no mostraron indicios de alteraciones moleculares, las concentraciones de $\mathrm{Cd} \mathrm{y} \mathrm{Pb}$ registrados en $T$. mactroides de Playa Caicara en la época de mayores precipitaciones constituyen una señal de alerta del ingreso de estos metales.

\section{REFERENCIAS}

Acosta V. y Lodeiros C. (2003). Índice ARN/ADN en poblaciones de la almeja Tivela mactroides (Bivalvia: Veneridae) provenientes de localidades con diferentes niveles de contaminación. Revista Científica de la Facultad de Ciencias Veterinarias de la Universidad del Zulia 13 (5), 378-382. 
Acosta V. y Lodeiros C. (2004a). Metales pesados en la almeja Tivela mactroides Born, 1778 (Bivalvia: Veneridae) en localidades costeras con diferentes grados de contaminación en Venezuela. Ciencias Marinas 30 (2), 1-11.

Acosta V. y Lodeiros C. (2004b). Efecto del cobre en juveniles de bivalvos (Tivela mactroides) provenientes de ambientes con diferentes niveles de contaminación. Boletín del Centro de Investigaciones Biológicas 38, 41-51.

Alfonso J.A., Azócar J.A., LaBrecque J.J., Benzo Z., Marcano E., Gómez C.V. y Quintal M. (2005). Temporal and spatial variation of trace metals in clams Tivela mactroidea along the Venezuelan coast. Mar. Pollut. Bull. 50, 1713-1744. https://doi.org/10.1016/j. marpolbul.2005.09.006.

ANVISA (2013). RDC 42, 29/08/2013. Regulamento técnico MERCOSUL sobre limites máximos de contaminantes inorgânicos em alimentos. Agência Nacional de Vigilância Sanitária. Diário Oficial da União, Brasil, 33 pp.

Arrieche D. y Prieto A. (2006). Parámetros poblacionales del guacuco Tivela mactroides (Bivalvia: Veneridae) de Playa Caicara, estado Anzoátegui, Venezuela. Ciencias Marinas 32 (2), 285-296. https://doi.org/10.7773/ cm.v32i21.1061

Azizi G., Akodad M., Baghour M., Layachi M. y Moumen A. (2018). The use of Mytilus spp. mussels as bioindicators of heavy metal pollution in the coastal environment: A review. J. Mater. Environ. Sci. 9 (4), 1170-1181. https://doi.org/10.26872/ jmes.2018.9.4.129

Bennedetti M., Giuliani M.E. y Regoli F. (2015). Oxidative metabolism of chemical pollutants in marine organisms: Molecular and biochemical biomarkers in environmental toxicology. Ann. NY Acad. Sci. 1340 (1), 8-19. https://doi.org/10.1111/nyas.12698

Bonilla J., Fermín J., Bamboa B. y Carrera M. (1995). Aspectos geoquímicos de los sedimentos superficiales del ecosistema marino costero de José, estado Anzoátegui, Venezuela. Boletín del Instituto Oceanográfico de Venezuela 34, 5-25.

Croquer A., Bone D., Bastidas, C. Ramos R. y García E. (2016). Monitoring coastal pollution associated with the largest oil refinery complex of Venezuela. Peer J. 4, e2171. https://doi.org/10.7717/peerj.2171

Ellman G.L. (1959). Tissue sulfhydryl groups. Arch. Biochem. Biophys. 82, 70-77. https://doi. org/10.1016/0003-9861(59)90090-6

EC (2017). Chemical safety. Council Regulation 315/93/ EEC: Commission Regulation. European Commission [en línea] https://ec.europa.eu/environment/chemicals/ index_en.htm 28/07/2019
FAO/WHO (2018). Evaluation of certain food additives and contaminants. Joint FAO/WHO Food Standards Programme Codex Committee on Contaminants in Foods. Food and Agriculture Organization of the United Nations/World Health Organization. Utrecht, Países Bajos, 169 pp.

Fernández A., Singh A. y Jaffe R. (2007). A literature review on trace metals and organic compounds of anthropogenic origin in the wider Caribbean region. Mar. Pollut. Bull. 54, 1681-1691. https://doi.org/10.1016/j. marpolbul.2007.08.007

Freites L., Lodeiros C., Arrieche D. y Dales A.W. (2014). Reproductive tactics of marine shellfish species from north-eastern Venezuela: Ecophysiological implications. En: Spawning: Biology, sexual strategies and ecological effects (Baqueiro-Cardenas E.R., Ed.). Nova Science, Nueva York, EUA, pp. 73-105.

Frías-Espericueta M.G., Osuna-López J.I., Voltolina D., López-López G., Izaguirre-Fierro G. y Muy-Rangel M.D. (2008). The metal content of bivalve molluscs of a coastal lagoon of NW Mexico. Bull. Environ. Contam. Toxicol. 80 (1), 90-92. https://doi.org/10.1007/ s00128-007-9322-4

Gagné F., André C. y Blaise C. (2008). The dual nature of metallothioneins in the metabolism of heavy metals and reactive oxygen species in aquatic organisms: implications of use as a biomarker of heavy-metal effects in field investigations. Biochem. Insights 1, 31-41. https://doi.org/10.4137/BCI.S1007

García E.M., Motta-Cruz J.J., Farina O. y Bastidas C. (2008). Anthropogenic influences on heavy metals across marine habitats in the western coast of Venezuela. Cont. Shelf Res. 28 (20), 2757-2766. https://doi. org/10.1016/j.csr.2008.09.020

Harris E.D. (1991). Copper transport: An overview. Proc. Soc. Exp. Med. 192, 130-140. https://doi. org/10.3181/00379727-196-43171b

Herrera A. y Bone D. (2011). Influence of riverine outputs on sandy beaches of Higuerote, central coast of Venezuela. Lat. Am. J. Aquat. Res. 39 (1), 56-70. https:// doi.org/10.3856/vol39-issue1-fulltext-6

Hook S.E., Gallagher E.P. y Batley G.E. (2014). The role of biomarkers in the assessment of aquatic ecosystem health. Integr. Environ. Assess. Manag. 10 (3), 327341. https://doi.org/10.1002/ieam.1530

INAMEH (2008). Boletín climatológico mensual. Instituto Nacional de Meteorología e Hidrología de Venezuela [en línea]. http://www.inameh.gob.ve/web/index.phpm 26/11/2009

Jaffé R., Leal I., Alvarado J., Gardinali P. y Sericanos J. (1995). Pollution effects of the Tuy river on the central Venezuelan coast: Anthropogenic organic compounds and heavy metal in Tivela mactroides. Mar. Pollut. 
Bull. 30, 290-300. https://doi.org/10.1016/0025326X(95)00087-4

Kang Y.J. (2006). Metallothionein redox cycle and function. Exp. Biol. Med. 231 (9), 1459-1467. https://doi. org/10.1177/153537020623100903

LaBrecque J., Benzo Z., Alfonso J., Cordovés P., Quintal M., Gómez C. y Marcano, E. (2004). The concentrations of select trace elements in clams, Tivela mactroidea along the Venezuelan coast in the state of Miranda. Mar. Pollut. Bull. 49, 659-667. https://doi. org/10.1016/j.marpolbul.2004.07.011

Lemus M., Laurent C., Acagua A., Cabrera M., Aponte A. y Chung K. (2010). Variación estacional de metales pesados en Perna viridis, de la localidad de Guayacán, península de Araya, estado Sucre, Venezuela. The Biologist 8 (2), 126-138.

Lemus M., Marín L., Aponte A. y Chung K. (2012). Metalotioninas, glutatión y consumo de oxígeno en el bivalvo Perna viridis expuesto a cadmio. Revista Científica de la Facultad de Ciencias Veterinarias de la Universidad del Zulia 22 (4), 376-380.

Lemus M., Salazar R., Lapo B. y Chung K. (2016). Metalotioneínas en bivalvos marinos. Lat. Am. J. Aquat. Res. 44 (2), 202-215. https://doi.org/10.3856/vol44issue2-fulltext-2

Lowry O., Rosebrough N., Farr J. y Randall R. (1951). Protein measurement with the Folin phenol reagent. J. Biol. Chem. 193 (1), 265-275.

Mao H., Wang D.H y Yang W.X. (2012). The involvement of metallothionein in the development of aquatic invertebrate. Aquat. Toxicol. 110 (111), 208-213. https:// doi.org/10.1016/j.aquatox.2012.01.018

Ohkawa H., Ohishi N. y Yagi K. (1979). Assay for lipid peroxides in animal tissues by thiobarbituric acid reaction. Rev. Anal. Biochem. 95 (2), 351-358. https://doi. org/10.1016/0003-2697(79)90738-3

Pan K. y Wang X.W. (2009). Biodynamics to explain the difference of copper body concentrations in five marine bivalve species. Environ. Sci. Technol. 43 (6): 21372143. https://doi.org/10.1021/es802888u

Pinto R., Acosta V., Segnini M.I., Brito L. y Martínez G. (2014). Temporal variations of heavy metals levels in Perna viridis, on the Chacopata-Bocaripo lagoon axis, Sucre State, Venezuela. Mar. Pollut. Bull. 91 (2): 41823. https://doi.org/10.1016/j.marpolbul.2014.09.059

Prieto A. (1983). Ecología de Tivela mactroides (Born, 1778) (Mollusca: Bivalvia) en la playa Güiria. Boletín del Instituto Oceanográfico de Venezuela 22 (1-2), 7-19.

Rabei A., Beldi H. y Soltani N. (2018). Multi-biomarker approach in transplanted Donax trunculus (Mollusc, Bivalve) from the Gulf of Annaba (Algeria). En: Recent advances in environmental science from the Euro-Mediterranean and surrounding regions, ad- vances in science, technology \& innovation (Kallel A., Ksibi M., Dhia H.B. y Khélifi N., Eds.). Springer International, Nueva York, EUA, 1449-1451. https:// doi.org/10.1007/978-3-319-70548-4_423

Regoli F. y Giuliani M.E. (2014). Oxidative pathways of chemical toxicity and oxidative stress biomarkers in marine organisms. Mar. Environ. Res. 93, 106-117. https://doi.org/10.1016/j.marenvres.2013.07.006

Rojas L., Chang I., Agard J., Bekele I. y Hubbard R. (2002). Heavy metals in green mussel (Perna viridis) and oysters (Crassostrea sp.) from Trinidad and Venezuela. Arch. Environ. Contam. Toxicol. 42, 410-415. https://doi.org/10.1007/s00244-001-0044-z

Ruttkay-Nedecky B., Nejdl L., Gumulec J., Zitka O., Masarik M, Eckschlager T., Stiborova M., Vojtech A. y Kizek R. (2013). The role of metallothionein in oxidative stress. Int. J. Mol. Sci. 14, 644-606. https:// doi.org/10.3390/ijms 14036044

Saad A.A., el-Sikaily A. y Kassem H. (2016). Metallothionein and glutathione content as biomarkers of metal pollution in mussels and local fishermen in Abu Qir Bay, Egypt. J. Health Pollut. 12, 50-60. https://doi. org/10.5696/2156-9614-6-12.50

Salman J.M., al-Mamoori A.M.J., Hughes R.A. y alSaadi A.H. (2013). Seasonal variations of biochemical markers in two species of molluscs as indication of heavy metals pollution. Int. J. Adv. Biol. Res. 3 (3), 1-5.

Sardi A. E., Ramos R. y García E. M. (2012). Hydrocarbon biomarkers responses in the bivalve, Tivela mactroides, exposed to polluted sediments. Bull. Environ. Contam. Toxicol. 90 (1), 1-8. https://doi.org/10.1007/s00128012-0862-x

Sedlak J. y Lindsay R. (1968). Estimation of total, proteinbound, and nonprotein sulfhydryl groups in tissue with Ellman's reagent. Anal. Biochem. 25, 192-205. https:// doi.org/10.1016/0003-2697(68)90092-4

Sokal R. y Rohlf J. (2012). Biometry: The principles and practice of statistics in biological research. 4a ed. WH Freeman, San Francisco, EUA, 935 pp. https://doi. org $/ 10.2307 / 2343822$

Viarengo A., Ponzano E., Dondero F. y Fabbri R. (1997). A simple spectrophotometric method for metallothionein evaluation in marine organisms: An application to Mediterranean and Antarctic molluscs. Mar. Environ. Res. 44, 69-84. https://doi.org/10.1016/S01411136(96)00103-1

Wang W.X. y Rainbow P.S. (2010). Significance of metallothioneins in metal accumulation kinetics in marine animals. Comp. Biochem. Physiol. C 152, 1-8. https://doi.org/10.1016/j.cbpc.2010.02.015

Wang W.X. y Guangyuan L. (2017). Heavy metals in bivalve mollusks. En: Chemical contaminants and residues in 
food (Cartus A. y Schrenk D., Eds.). Elsevier, Ámsterdam, Países Bajos, 553-594 (Woodhead Publishing Series in Food Science, Technology and Nutrition). https://doi.org/10.1016/B978-0-08-100674-0.00021-7

Wang W.X., Meng J. y Nanyan W. (2018). Metals in oysters: Molecular and cellular mechanisms and ecotoxicological impacts. Environ. Sci.-Proc. Imp. 20 (6), 892-912. https://doi.org/10.1039/C8EM00069G

Zapata-Vívenes E. y Nusetti O. (2007). Protection of glycolytic enzymes by metallothioneins from oxidative damage in the digestive gland of green lipped mussel Perna viridis. J. Shell. Res. 26 (2), 1-10. https://doi. org/10.2983/0730-8000(2007)26[335:POGEBM]2. $0 . \mathrm{CO} ; 2$

Zapata-Vívenes E., Rojas de Astudillo L., Sánchez G. y Barreto M. (2012). Heavy metals and related biomarkers in Perna viridis (Bivalvia: Mytilidae) collected on the coast of Sucre State, Venezuela. Ciencias Marinas 38 (3), 517-528. https://doi.org/10.7773/ cm.v38i3.2046 